\title{
The Predictive Ability of Two Triglyceride-Associated Indices for Gestational Diabetes Mellitus and Large for Gestational Age Infant Among Chinese Pregnancies: A Preliminary Cohort Study
}

This article was published in the following Dove Press journal:

Diabetes, Metabolic Syndrome and Obesity: Targets and Therapy

\author{
Peng Ju Liu' \\ Yanping Liu $\mathbb{1}^{1}$ \\ Liangkun $\mathrm{Ma} \mathbb{D}^{2}$ \\ Ai Min $\mathrm{Yao}^{3}$ \\ Xiao Yan Chen ${ }^{4}$ \\ Yi Xuan $\mathrm{Hou}^{5}$ \\ Li Ping $W^{5}$ \\ Liang Yu $\mathrm{Xia}^{6}$
}

'Department of Clinical Nutrition, Peking Union Medical College Hospital, China Academic Medical Science and Peking Union Medical College, Beijing, People's Republic of China; ${ }^{2}$ Department of Gynaecology and Obstetrics, Peking Union Medical College Hospital, China Academic Medical Science and Peking Union Medical College, Beijing, People's Republic of China; ${ }^{3}$ Department of Gynaecology and Obstetrics, Shunyi District Maternal and Child Health Hospital, Beijing, People's Republic of China; ${ }^{4}$ Department of Gynaecology and Obstetrics, Quanzhou Maternal and Child Health Hospital, Fujian, People's Republic of China; ${ }^{5}$ Peking Union Medical College School of Nursing, Beijing, People's Republic of China; ${ }^{6}$ Department of Clinical Laboratory, Peking Union Medical College Hospital, Peking Union Medical College Hospital, China Academic Medical Science and Peking Union Medical College, Beijing, People's Republic of China
Correspondence: Yanping Liu

Tel +86-|0-69|5908|

Fax +86-10-69155551

Email liuyp1227@vip.sina.com
Background/Aims: To investigate the potential of maternal first-trimester triglyceride (TG) to high-density lipoprotein cholesterol (TG/HDL-c) ratio, triglyceride glucose index (TyG) and total cholesterol (TC)/HDL-c to predict the risk of later gestational diabetes mellitus (GDM) and large for gestational age (LGA) newborn in Chinese women.

Methods: We included 352 women with a singleton pregnancy, who were followed up prospectively from the first prenatal visit until delivery. Fasting glucose and plasma lipid profiles including TG, TC, HDL-c, and low-density lipoprotein cholesterol (LDL-c) were measured in the first trimester. A binary logistic regression analysis was performed to determine the odds ratios (ORs) and 95\% confidence intervals (CIs) of GDM and LGA according to tertiles of those indices, respectively. Receiver-operating characteristic curve (ROC) and areas under the curve (AUC) were employed to evaluate the ability of those indices to predict the risk of GDM and LGA infants, and differences in the AUC values between them were compared.

Results: Women with the top tertile of TG/HDL-c or TyG other than TC/HDL-c had a significantly higher risk of GDM $\left(\mathrm{OR}_{\mathrm{TG} / \mathrm{HDL}-\mathrm{c}}=2.388,95 \%\right.$ CI $1.026-5.467 ; \mathrm{OR}_{\mathrm{TyG}}$ $=3.535$, 95\% CI 1.483-8.426, respectively) and LGA infant delivery $\left(\mathrm{OR}_{\mathrm{TG} / \mathrm{HDL}-\mathrm{c}}=3.742\right.$, $95 \%$ CI $1.114-12.569 ; \mathrm{OR}_{\mathrm{TyG}}=3.011,95 \%$ CI $1.012-8.962$, respectively) than women with the lowest tertile of TG/HDL-c or TyG after adjusting for confounders. The AUC of TG/ HDL-c and TyG to detect GDM was 0.664 (95\% CI $0.595-0.733)$ and $0.686(95 \% \mathrm{CI}$ $0.615-0.756)$, respectively, and that to detect LGA was 0.646 (95\% CI $0.559-0.734)$ and 0.643 (95\% CI 0.552-0.735), respectively (all $\mathrm{P}<0.01$ ). There were no statistical differences between TG/HDL-c and TyG in the ability of predicting the risk of GDM or LGA infants. Conclusion: Maternal first-trimester TG/HDL-c and TyG are both good indicators in predicting the risk of later GDM and LGA newborn, and it may be useful to evaluate them in early pregnancy.

Keywords: gestational diabetes, triglyceride/HDL-c ratio, triglyceride glucose index, large for gestational age infant

\section{Introduction}

Gestational diabetes mellitus (GDM) is currently the most common medical complication of pregnancy. The documented prevalence of GDM varies substantially worldwide, ranging from $1 \%$ to $30 \%{ }^{1}$ Due to a lack of consensus and consistency in the screening and diagnostic criteria for GDM, it is challenging to compare the 
prevalence across countries and regions. ${ }^{1}$ According to one most recent systematic review and meta-analysis, in which the International Association of Diabetes and Pregnancy Study Groups (IADPSG) criteria was used as the diagnostic criteria of GDM, the total incidence of GDM in mainland China can reach approximately $15 \%{ }^{2}$ GDM is defined as any degree of glucose intolerance that was first recognized during pregnancy. ${ }^{3,4}$ Many factors are considered to be risk factors for GDM, including mother's ethnicity, body composition, family history of diabetes, obesity, and polycystic ovary syndrome (PCOS). ${ }^{5,6}$ GDM has a variety of negative implications for mothers and their offspring. For mothers, GDM is associated with higher rates of preeclampsia, cesarean deliveries, shoulder dystocia, and type 2 diabetes mellitus in the postpartum period. ${ }^{5,7}$ On the other side, it is evident that the women with GDM are more likely to deliver large for gestational age (LGA) infant than nondiabetic mothers, which is an important determinant factor in perinatal morbidity and mortality. ${ }^{8}$ Furthermore, offspring born to mothers with GDM have a higher likelihood of developing obesity and of having impaired glucose tolerance (IGT) as well as type 2 diabetes in childhood or in early adulthood. ${ }^{5,9,10}$ Being overweight or obese or having IGT is one of significant risk factors for GDM, and this gives rise to a vicious intergenerational cycle of obesity and diabetes. Therefore, early prediction GDM may help in improved clinical management of pregnancy.

Pregnancy is a state of multiple and critical changes in the morphology and physiology of women, which play a fundamental role in meeting the mother's basal needs and the requirements of the developing fetus. ${ }^{11}$ Among the maternal physiological adaptations, alterations in lipid metabolism are more characteristic. ${ }^{12,13}$ The first 2 trimesters of pregnancy are an anabolic phase, which is attributed to several factors that cooperatively increase the deposition of fat in maternal tissues, ${ }^{12,14}$ including maternal hyperphagia, enhanced lipogenesis, and decreased adipose tissue lipolytic activity. As a result, those maternal factors result in net triglyceride accumulation. ${ }^{12}$ Along with the changes of adipose tissue during pregnancy, serum triglycerides (TG), lipoproteins and cholesterol alter as well. In a large cross-sectional study, TG, total cholesterol (TC) and low-density lipoprotein cholesterol (LDL-c) levels decreased between the time of conception and 8 weeks of gestation and then gradually increased and peaked just before delivery. ${ }^{15}$ In a prospective cohort study, the mean serum concentrations of those lipids including TG, TC, and LDL increased from the first trimester to the third trimester. ${ }^{16}$ Increasing evidence has suggested that hypertriglyceridemia, even in early pregnancy, ${ }^{17}$ is likely to be associated with insulin resistance, ${ }^{18,19}$ as well as GDM. ${ }^{20,21}$ In addition, fasting plasma glucose (FPG) at the first prenatal visit is related to glycemic metabolism in the mid-gestational period, as suggested in a previous study. ${ }^{22}$ Moreover, a recent study has reported that high FPG or BMI in the first trimester of pregnancy is independently associated with later development of GDM. ${ }^{23}$ Therefore, it is suggested that the evaluation of maternal first-trimester serum concentrations of TG, lipoproteins, cholesterol and FPG may be of great significance for the prediction of complications in later pregnancy.

Recently, two triglyceride-associated markers, ratio of TG to high-density lipoprotein cholesterol (TG/HDL-c) and triglyceride glucose index (TyG), have been proposed as indicators of insulin resistance, ${ }^{24-27}$ which is the principal pathophysiology of GDM. ${ }^{6}$ Not only in the general population, but also in pregnant women, these two indices have been reported to be useful in monitoring insulin resistance during pregnancy ${ }^{28}$ as well as in predicting the risk of GDM and LGA newborn. ${ }^{29,30}$ However, it is still unclear whether these indices have the same or different potential in predicting GDM or LGA infant delivery.

The aim of this study was to evaluate the associations of these two TG-associated indices as well as TC/HDL-c of early pregnancy with the risk of developing GDM and delivering LGA newborn, using data from a motheroffspring cohort study where the correlation between the single nucleotide polymorphism (SNP) of the nutrientassociated genes and the maternal nutrient status were investigated.

\section{Materials and Methods}

\section{Ethical Statement}

The study protocol was approved by the Ethics Committee of Peking Union Medical College Hospital of the Chinese Academy of Medical Science (Date of approval: 24-July -2018; ref. no., hs-1646). This study was conducted in accordance with both the Declaration of Helsinki of 1975, as revised in 1983, and guidelines of the centre's institutional review board. All the participants were informed of the details of the study, and each participant provided written informed consent. 


\section{Participants}

The recruitment of participants in this cohort took place during October and December 2018 at the Shunyi District Maternal and Child Health Hospital (Beijing, PR China), where women in early pregnancy were approached to participate. All the women are local residents in Shunyi District of Beijing, and they had established prenatal records before recruitment. At first, a total of 432 pregnant women agreed to participate in this cohort. A standard questionnaire was used by trained researchers to collect information, including age, ethnicity (self-report), smoking status, drinking status, education (senior middle school or lower/college degree or higher), physical activity (0150 mins or $\geq 150$ mins moderate exercise time per week), parity (primiparous or multiparous), medical history, family history of diabetes (yes/no). Women were excluded if they had any of the following criteria: (1) not a singleton pregnancy; (2) those who are not the Han ethnicity (to avoid possible confounding by ethnicity); (3) those who lab-testing fasting glucose $\geq 6.1 \mathrm{mmol} / \mathrm{L}$ and/or $\mathrm{HbAlc}>$ $6.5 \%$ or diagnosed as diabetes before pregnancy; (4) those who with a history of autoimmune disease (such as systemic lupus erythematosus, etc) or currently use corticosteroids; (5) those who with define hyperthyroidism or hypothyroidism; (6) those who miscarried or induced labor before the 75 -g oral glucose tolerance test (OGTT) at 24-28 weeks' gestation; (7) those who with a history of liver or renal insufficiency or with presumed acute inflammation (CRP $>10 \mathrm{mg} / \mathrm{L})$; (8) those who with a suspected familial hypertriglyceridemia (serum $\mathrm{TG} \geq 5.1 \mathrm{mmol} / \mathrm{L}$ ); (9) those who without complete records of lipid profiles and/or FPG concentration.

Height was measured to nearest $0.1 \mathrm{~cm}$ with a portable stadiometer. Weight was measured in an upright position to the nearest $0.1 \mathrm{~kg}$ with a calibrated scale. Body mass index (BMI) was calculated as weight $(\mathrm{kg}) /$ height $(\mathrm{m}){ }^{2}$ During the whole course of pregnancy, the routine prenatal examination for each participant was performed in the same hospital. At 24-28 weeks' gestation, a 75-g OGTT was conducted in all participants. The diagnosis of GDM was using IADPSG/WHO criteria where one or more glucose values from a 75-g OGTT equaled or exceeded the following thresholds: FPG $5.1 \mathrm{mmol} / \mathrm{L}, 1-\mathrm{h}$ plasma glucose (PG) $10.0 \mathrm{mmol} / \mathrm{L}$, and $2-\mathrm{h}$ PG $8.5 \mathrm{mmol} / \mathrm{L}^{31}$ After delivery, all the related data were sourced from medical records. LGA was defined as birthweight $>90$ th percentile for gestational age and sex. ${ }^{32}$ Finally, a total of 352 women with completed data were eligible for this study. The participant flowchart was presented in Figure 1.

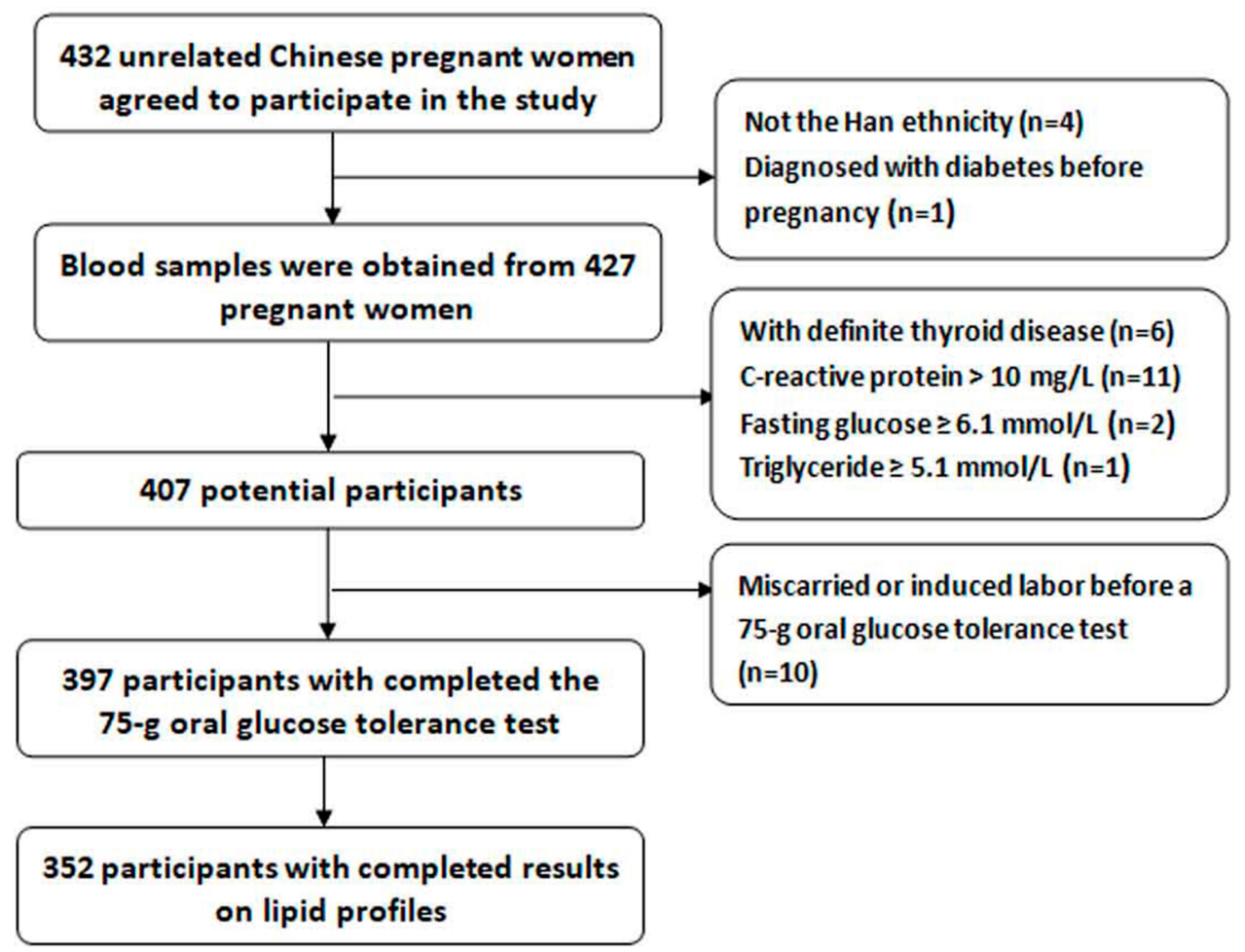

Figure I Participant flowchart. 


\section{Measurements}

Blood samples were collected from the peripheral vein of participants during the first visit before 12 weeks' gestation, after an overnight (at least 8 hours) fast. Maternal FPG, TC, TG, HDL-c, LDL-c, and CRP concentrations during the first trimester of pregnancy were measured by an automatic analyser (Beckman 5800; Beckman Coulter Inc., Brea, CA, USA) and retrieved from medical records. Homeostasis model assessment-insulin resistance (HOMA-IR) was calculated as follows:

HOMA-IR $=($ fasting plasma glucose $[\mathrm{mmol} / \mathrm{L}] \times$ fasting serum insulin $[\mu \mathrm{IU} / \mathrm{L}]) / 22.5$

TyG was calculated as: $\mathrm{TyG}=\mathrm{Ln}[\mathrm{TG}(\mathrm{mg} / \mathrm{dl}) \mathrm{FPG}$ $(\mathrm{mg} / \mathrm{dl}) / 2]^{33}$

\section{Statistical Analysis}

Statistical analyses were performed using the Statistical Software Package for Social Science (SPSS version 16.0, Chicago, IL, USA). Normally distributed variables were presented as mean (standard deviation), while skewed variables were presented as median (interquartile range, 25-75\%). Categorical variables were described as frequency or percentage and examined by the Chi square test. The independent-sample $t$-test or the Mann-Whitney $U$-test, when appropriate, was used to compare variables with normal or skewed distribution between the GDM group and the non-GDM group. Spearman correlations were applied to assess the correlations between the indices and variables including age, BMI, HOMA-IR, and CRP. TG/HDL-c (T1, < 0.49; T2, 0.49-0.74; T3, $\geq 0.75)$, TyG (T1, < 7.9; T2, 7.9-8.3; T3, $\geq 8.3$ ), and TC/HDL-c (T1, < 2.5; T2, 2.5-2.9; T3, $\geq$ 2.9) were divided into tertiles according to their respective cut-off points of entire distribution for this study population. Binary logistic regression analyses were performed to determine the odds ratio (ORs) and 95\% confidence intervals (CIs) of GDM and LGA according to respective tertiles of the three indices with adjusting for potential covariates. Receiving-operating characteristic (ROC) curves and areas under the curve (AUC) were employed to evaluate the ability of the indices to detect GDM and LGA newborn. A comparison of the diagnostic abilities between the indices was performed using the AUC, and the significance of differences between their AUC values was also assessed using the method described by DeLong. ${ }^{34}$

\section{Results}

\section{Maternal and Infant Characteristics}

In our study, none of the women smoke or drink for at least three months before enrolment, thus we did not show the data on smoking and drinking status. The prevalence of GDM was $18.8 \%(n=66)$ in this study. Compared with women without GDM, women with GDM had significantly higher age, BMI (at enrolment), TG, LDL-c, FPG, HOMA-IR, CRP, TG/HDL-c, TyG, TC/HDL-c, and percentage of family history of diabetes, but a less $(P=0.017)$ exercise time per week, a lower trend in HDL-c $(P=0.058)$ and a higher trend in the incidence of cesarean section $(P=0.068)$. No significant differences in parity, education, weight gain and percentage of history of PCOS were found between women with and without GDM (Table 1).

On the neonate side, the overall prevalence of LGA was $8.8 \%(n=31)$, and there was a significant difference in the prevalence of LGA in women with and without GDM $(P=0.001)$, and there were no significant differences between groups in gestational age, birth weight and infant sex (Table 1).

\section{Correlations Between the}

\section{Triglyceride-Associated Indices and Markers of Inflammation and Insulin Resistance}

Spearman correlation analysis showed that all the indices were significantly correlated with maternal age, BMI (at enrolment), HOMA-IR, and CRP (all $P<0.01$, Table 2). In addition, there was a high correlation among the three indices $(P<0.001)$.

\section{Associations Between the Tertiles of the Two Indices and the Risk of Developing GDM and Delivering LGA Infant}

Values of TG/HDL-c, TyG index, and TC/HDL-c were divided into tertiles and their lowest tertiles were used as reference, respectively. Then, binary logistic regression analyses were used to explore the associations between the quartiles of the two indices and GDM risk, respectively (Table 3). Women in the upper two tertiles of TC/HDL-c values did not have a significantly higher risk of GDM or LGA delivery than those in the lowest tertile after adjustment for covariates (all $p>0.05$ ). 
Table I Maternal and Infant Characteristics

\begin{tabular}{|c|c|c|c|c|}
\hline Variables & All $(n=352)$ & GDM $(n=66)$ & Non-GDM $(n=286)$ & $P$ \\
\hline $\begin{array}{l}\text { Maternal characteristics } \\
\text { Age (years) } \\
\text { Body mass index }\left(\mathrm{kg} / \mathrm{m}^{2}\right) \\
\text { Weight gain }(\mathrm{kg})^{\mathrm{a}} \\
\text { Weight gain }(\mathrm{kg})^{\mathrm{b}} \\
\text { Total cholesterol }(\mathrm{mmol} / \mathrm{L}) \\
\text { Triglyceride }(\mathrm{mmol} / \mathrm{L}) \\
\text { HDL-c }(\mathrm{mmol} / \mathrm{L}) \\
\text { LDL-c }(\mathrm{mmol} / \mathrm{L}) \\
\text { TG/HDL-c ratio } \\
\text { TC/HDL-c ratio } \\
\text { Fasting plasma glucose }(\mathrm{mmol} / \mathrm{L}) \\
\text { Triglyceride glucose index } \\
\text { HOMA-IR } \\
\text { C-reactive protein }(\mathrm{mg} / \mathrm{L}) \\
\text { Family history of DM }\end{array}$ & $\begin{array}{l}29.2(3.6) \\
22.8(3.7) \\
9.3(4.6) \\
15.1(5.8) \\
4.20(0.69) \\
0.93(0.72-1.21) \\
1.54(0.28) \\
2.0(0.54) \\
0.60(0.46-0.85) \\
2.71(2.42-3.09) \\
4.5(4.2-4.7) \\
8.1(0.5) \\
1.52(1.05-2.29) \\
2.1(0.9-4.0) \\
31(8.8)\end{array}$ & $\begin{array}{l}30.4(4.0) \\
24.3(3.6) \\
9.4(4.4) \\
14.1(6.7) \\
4.29(0.71) \\
1.10(0.87-1.46) \\
1.49(0.26) \\
2.15(0.58) \\
0.73(0.55-1.05) \\
2.88(2.55-3.27) \\
4.7(4.4-5.0) \\
8.3(0.4) \\
2.11(1.40-3.07) \\
3.3(1.5-5.9) \\
11(16.7)\end{array}$ & $\begin{array}{l}29.0(3.5) \\
22.4(3.6) \\
9.0(5.5) \\
15.3(5.5) \\
4.17(0.68) \\
0.90(0.71-1.15) \\
1.56(0.28) \\
1.96(0.53) \\
0.58(0.44-0.80) \\
2.65(2.37-3.02) \\
4.4(4.1-4.7) \\
8.0(0.4) \\
1.40(1.00-2.08) \\
1.9(0.8-3.5) \\
20(7.0)\end{array}$ & $\begin{array}{l}0.007 \\
<0.001 \\
0.078 \\
0.224 \\
0.194 \\
<0.001 \\
0.058 \\
0.013 \\
<0.001 \\
0.002 \\
<0.001 \\
<0.001 \\
<0.001 \\
<0.001 \\
0.012\end{array}$ \\
\hline $\begin{array}{l}\text { Parity } \\
\text { Primiparous } \\
\text { Multiparous } \\
\text { History of PCOS }\end{array}$ & $\begin{array}{l}209(59.4) \\
143(40.6) \\
6(1.7)\end{array}$ & $\begin{array}{l}37(56.1) \\
29(43.9) \\
2(3.0)\end{array}$ & $\begin{array}{l}172(60.1) \\
114(39.9) \\
4(1.4)\end{array}$ & $\begin{array}{l}0.543 \\
0.356\end{array}$ \\
\hline $\begin{array}{l}\text { Education } \\
\text { Senior middle school or lower } \\
\text { College degree or higher }\end{array}$ & $\begin{array}{l}73(20.7) \\
279(79.3)\end{array}$ & $\begin{array}{l}12(18.2) \\
54(81.8)\end{array}$ & $\begin{array}{l}61(21.3) \\
225(78.7)\end{array}$ & 0.570 \\
\hline $\begin{array}{l}\text { Physical activity } \\
0-150 \text { mins per week } \\
\geq 150 \text { mins per week } \\
\text { Cesarean section }\end{array}$ & $\begin{array}{l}188(53.4) \\
164(46.6) \\
106(30.1)\end{array}$ & $\begin{array}{l}44(66.7) \\
22(33.3) \\
26(39.4)\end{array}$ & $\begin{array}{l}\text { I } 44(50.3) \\
142(49.7) \\
80(28.0)\end{array}$ & $\begin{array}{l}0.017 \\
0.068\end{array}$ \\
\hline $\begin{array}{l}\text { Infant characteristics } \\
\text { Gestational age } \\
\text { Sex (male) } \\
\text { Birth weight (g) } \\
\text { LGA }\end{array}$ & $\begin{array}{l}39(38-40) \\
191(54.3) \\
3369(476) \\
31(8.8)\end{array}$ & $\begin{array}{l}39(38-40) \\
38(57.6) \\
34 \mid 2(595) \\
13(19.7)\end{array}$ & $\begin{array}{l}39(38-40) \\
153(53.5) \\
3359(447) \\
18(6.3)\end{array}$ & $\begin{array}{l}0.114 \\
0.549 \\
0.512 \\
0.001\end{array}$ \\
\hline
\end{tabular}

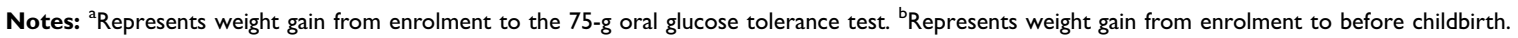

Abbreviations: TG, triglyceride; HDL-c, high-density lipoprotein cholesterol; LDL-c, low-density lipoprotein cholesterol; FBG, fasting blood glucose; HOMA-IR, homeostasis model assessment-insulin resistance; DM, diabetes mellitus; PCOS, polycystic ovary syndrome; LGA, large for gestational age.

For TG/HDL-c, compared to women in the lowest tertile, women in the highest tertile had a higher risk of developing GDM [adjusted OR=2.388, 95\% CI (1.026-5.467), $P<$ $0.05]$ after adjusting for covariates, including maternal age, $\mathrm{BMI}$ at enrolment, weight gain (from enrolment to the $75 \mathrm{-g}$ OGTT), education, physical activity, parity, family history of diabetes, history of PCOS, and CRP, and they also had a significantly higher risk of delivering LGA infants [adjusted OR=3.742, 95\% CI (1.114-12.569), $P<0.05$ ] after labor method, fetal sex, gestation age, and weight gain (from enrolment to before childbirth) were further adjusted following above maternal covariates.
As for TyG, compared with women in the reference tertile, women in the highest tertile also had a significantly higher risk of developing GDM [adjusted $\mathrm{OR}=3.535,95 \%$ CI (1.483-8.426), $P<0.01]$ and delivering LGA infants [adjusted $\mathrm{OR}=3.011,95 \%$ CI $(1.012-8.962), P<0.05$ ] after adjusting for the same covariates as above, respectively.

However, the associations between the two indices and the risk of LGA infant became non-significant when GDM (yes/no) was further adjusted as a cofounding factor following the same covariates as above in the regression models ( $\underline{\text { Supplemental Table)}}$. 
Table 2 Spearman Correlation Between the Indices and Age, BMI and Markers of Inflammation and Insulin Resistance

\begin{tabular}{|l|l|l|l|l|l|l|}
\hline \multirow{2}{*}{ Variables } & \multicolumn{2}{l|}{ TG/HDL-c } & \multicolumn{2}{l|}{ TyG } & \multicolumn{2}{l|}{ TC/HDL-c } \\
\cline { 2 - 7 } & Coefficient & $P$ & Coefficient & $P$ & \multicolumn{2}{l|}{ Coefficient } \\
\hline Age (years) & 0.17 & $<0.01$ & 0.21 & $<0.01$ & 0.14 & $P$ \\
BMI $\left(\mathrm{kg} / \mathrm{m}^{2}\right)$ & 0.40 & $<0.01$ & 0.39 & $<0.01$ & 0.43 & $<0.01$ \\
HOMA-IR & 0.39 & $<0.01$ & 0.43 & $<0.01$ & 0.37 & $<0.01$ \\
CRP $(\mathrm{mg} / \mathrm{L})$ & 0.46 & $<0.01$ & 0.53 & $<0.01$ & 0.28 & $<0.01$ \\
\hline
\end{tabular}

Abbreviations: TG, triglyceride; HDL-c, high-density lipoprotein cholesterol; HOMA-IR, homeostasis model assessment-insulin resistance; CRP, C-reactive protein; TyG, triglyceride glucose index; TC, total cholesterol.

Table 3 Associations Between Tertiles of the Indices and the Risk of GDM and LGA Infants

\begin{tabular}{|c|c|c|c|c|}
\hline \multirow[t]{2}{*}{ Tertiles of Indices } & \multirow[b]{2}{*}{ GDM (n) } & \multirow[t]{2}{*}{ LGA (n) } & \multicolumn{2}{|c|}{ Adjusted Odds Ratio ( $95 \%$ Confidence Interval) } \\
\hline & & & GDM $^{\dagger}$ & LGA Infant $\ddagger$ \\
\hline \multicolumn{5}{|l|}{ TG/HDL-c } \\
\hline Tertile I & 11 & 4 & Reference & Reference \\
\hline Tertile 2 & 22 & 10 & $1.862(0.804-4.313)$ & $2.475(0.722-8.482)$ \\
\hline Tertile 3 & 33 & 17 & $2.388(1.026-5.467)^{*}$ & $3.742(1.114-12.569)^{*}$ \\
\hline \multicolumn{5}{|l|}{ TyG index } \\
\hline Tertile I & 10 & 6 & Reference & Reference \\
\hline Tertile 2 & 19 & 7 & $2.273(0.959-5.387)$ & $1.057(0.325-3.431)$ \\
\hline Tertile 3 & 37 & 18 & $3.535(1.483-8.426)^{* *}$ & $3.011(1.012-8.962)^{*}$ \\
\hline \multicolumn{5}{|l|}{ TC/HDL-C } \\
\hline Tertile I & 14 & 6 & Reference & Reference \\
\hline Tertile 2 & 21 & 7 & $1.26 \mid(0.578-2.750)$ & $1.085(0.342-3.442)$ \\
\hline Tertile 3 & 31 & 18 & $1.683(0.772-3.666)$ & $2.584(0.893-7.362)$ \\
\hline
\end{tabular}

Notes: ${ }^{\dagger}$ adjusted for age, education, physical activity, BMI (at enrolment), parity, family history of diabetes, history of PCOS, CRP and weight gain (from enrolment to the 75$\mathrm{g}$ oral glucose tolerance test); ładjusted for age, education, physical activity, BMI (at enrolment), parity, family history of diabetes, history of PCOS, CRP, labor method, fetal sex, gestation age and weight gain (from enrolment to before childbirth); ${ }^{*} p<0.05,{ }^{* *} p<0.01$.

Abbreviations: TG, triglyceride; HDL-c, high-density lipoprotein cholesterol; TC, total cholesterol.

\section{ROC Curve Analyses of}

Triglyceride-Associated Indicators of GDM Development and LGA Infant

\section{Delivery}

The AUC values for detecting GDM and LGA infant by TG/HDL-c and TyG, as well as HOMA-IR, are shown in Table 4. As for the detection of GDM, all three indices showed significant areas under the ROC curve $(P<0.001$ for all, Table 4 and Figure 2). Among of them, the AUC value and $95 \% \mathrm{CI}$ for TyG was almost the same as that for HOMA-IR, but was slightly higher than that for TG/HDLc. Further, the differences in AUC values among the three indices were also compared. According to the method described by DeLong, ${ }^{34}$ there were no significant differences among them (TG/HDL-c vs HOMA-IR, $z=1.878$, $P=0.06$; TyG vs HOMA-IR, $\mathrm{z}=0.019, P=0.985$; TyG vs TG/HDL-c, $\mathrm{z}=0.515, P=0.607$ ).
The AUC of TG/HDL-c, TyG index and HOMA-IR to detect LGA infant delivery was 0.646 (95\% CI, 0.559-$0.734, P=0.007), 0.643$ (95\% CI, 0.552-0.735, $P=0.008$ ) and 0.603 (95\% CI, 0.511-0.696, $P=0.057)$, respectively (Table 4 and Figure 3). Likewise, differences in AUC values between the two triglyceride-associated indices were also compared. According to the above method, there were no significant differences among them (TyG vs TG/HDL-c, $\mathrm{z}=0.0992, P=0.921)$.

\section{Discussion}

The present study showed that women who developed GDM had significantly increased TG/HDL-c ratios and TyG values in early pregnancy compared with controls; women with TG/HDL-c ratios or TyG values in the top tertile had a significantly higher risk of developing GDM and delivering LGA infants than did those with TG/HDL-c 
Table 4 Area Under Curve (AUC) and Corresponding 95\% Confidence Interval (Cl) of the Two Indices and HOMA-IR for Detecting GDM Development and LGA Infant Delivery

\begin{tabular}{|l|l|l|l|l|l|l|}
\hline \multirow{2}{*}{ Variables } & \multicolumn{2}{l|}{ Gestational Diabetes Mellitus } & \multicolumn{3}{l|}{ Large for Gestational Age Infant } \\
\cline { 2 - 7 } & SE & AUC (95\% CI) & $P$ & SE & AUC (95\% CI) \\
\hline HOMA-IR & 0.35 & $0.685(0.614-0.756)$ & $<0.001$ & 0.047 & $0.603(0.511-0.696)$ & 0.057 \\
TyG & 0.36 & $0.686(0.615-0.756)$ & $<0.001$ & 0.047 & $0.643(0.552-0.735)$ & 0.008 \\
TG/HDL-c & 0.35 & $0.664(0.595-0.733)$ & $<0.001$ & 0.045 & $0.646(0.559-0.734)$ \\
\hline
\end{tabular}

Abbreviations: SE, standard error; HOMA-IR, homeostasis model assessment-insulin resistance; TyG, triglyceride glucose index; TG, triglyceride; HDL-c, high-density lipoprotein cholesterol.

ratios or TyG values in the lowest tertile, suggesting that both TG/HDL-c and TyG at the time of the first trimester of pregnancy are independently and significantly associated with the later development of GDM and LGA infant delivery, which strengthens previous findings. ${ }^{28-30}$ There were no statistical differences between TG/HDL-c and TyG in the ability of predicting the risk of GDM or LGA.

The overall incidence of GDM (18.8\%) in this study was slightly higher than that reported in a recent metaanalysis where the average incidence of GDM was estimated to be $15 \% .^{2}$ Possible explanations might include: 1 ) more than $50 \%$ of pregnant women in the study population have less than 150 minutes of moderate exercise per week; 2) women with BMI $\geq 24.0 \mathrm{~kg} / \mathrm{m}^{2}$ at the time of enrolment accounted for $30.1 \%(\mathrm{n}=109)$ of the study population. These factors might be associated with the high incidence of GDM.

An elevated serum level of TGs is common in pregnant women, especially in GDM pregnancies, and this may be associated with insulin resistance, relative lack of insulin secretion and oxidative stress. ${ }^{29}$ Several studies have found that hypertriglyceridemia, even in early pregnancy, ${ }^{17}$ is associated with insulin resistance as well as GDM. ${ }^{18-21}$ Enquobahrie et al found that each $20 \mathrm{mg} / \mathrm{dl}$ increase in $\mathrm{TG}$ concentration leads to a $10 \%$ rise in the risk of GDM. Also, they demonstrated that mothers with

\title{
ROC Curve
}

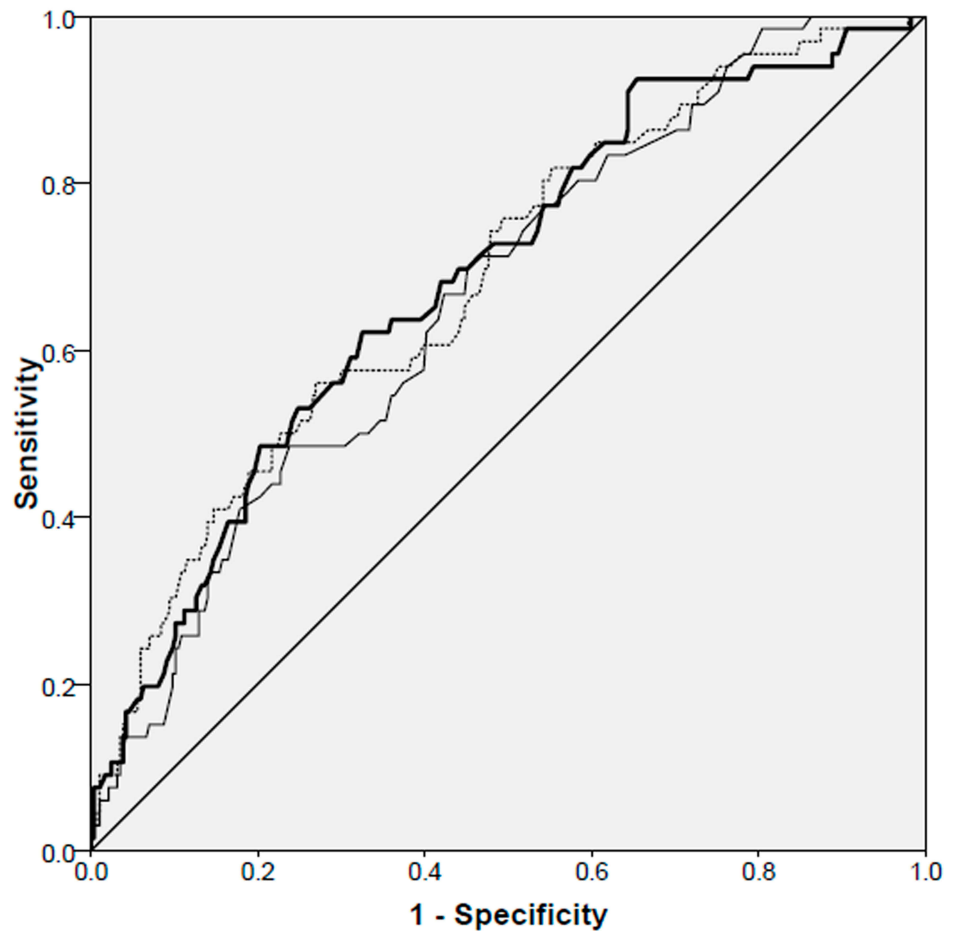

\author{
Source of the Curve \\ -TyG \\ -...-HOMA-IR \\ -TG/HDL-c \\ -Reference Line
}

Figure 2 Receiver operating characteristic (ROC) curves of triglyceride-associated indices and HOMA-IR used to predict GDM development. 
ROC Curve

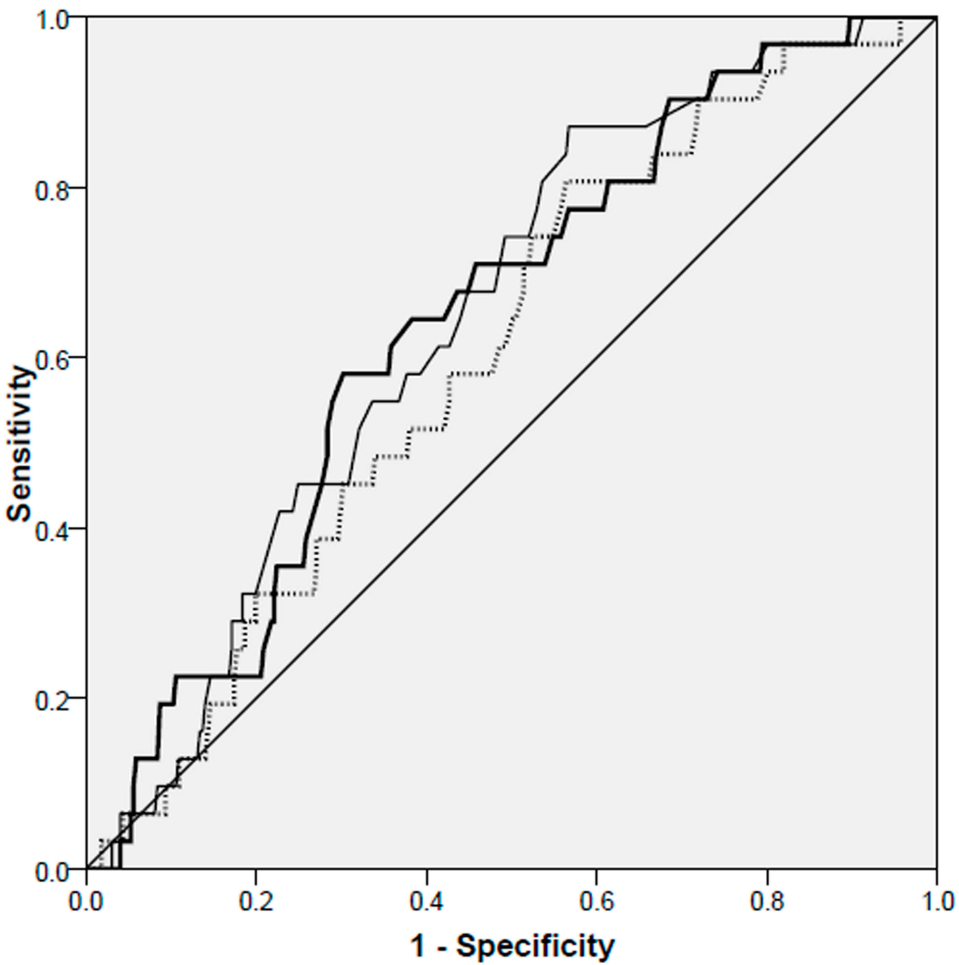

Source of the Curve

-TyG

-.'HOMA-IR

$-\mathrm{TG} / \mathrm{HDL}-\mathrm{C}$

-Reference Line

Figure 3 Receiver operating characteristic (ROC) curves of triglyceride-associated indices and HOMA-IR used to predict LGA infant delivery.

TG levels more than $137 \mathrm{mg} / \mathrm{dl}$ were 3.5 times more likely to develop GDM. ${ }^{35}$ Furthermore, it has been confirmed that the level of maternal TGs has an independent and strong association with birth weight, whether women have GDM or not. ${ }^{36-39}$ There are some pathophysiological reasons for the increased risk of macrosomia in pregnant women with hypertriglyceridemia. Serum level of TGs is subject to significant changes in pregnancy trimesters. In the first trimester of pregnancy, insulin sensitivity and lipoprotein lipase activity increase. Conversely, the lipoprotein lipase activity decreases in the third trimester of pregnancy due to the increase in insulin resistance, a phenomenon which is more prominent in GDM. ${ }^{40}$ The derived fatty acids enter the umbilical cord blood, are stored in fetal adipose tissues, and result in increased fetal growth and adiposity. ${ }^{41}$ In addition, the serum concentration of HDL-c has been reported to be negatively associated with the risk for GDM and macrosomia and a moderate rise in concentration of HDL-c is a protective factor for both of them. ${ }^{42}$ Therefore, TG/HDL-c, an index combined of TG and HDL-c, has been demonstrated to be associated with both GDM and LGA infant delivery, ${ }^{29}$ as reported by Wang et al, the area under ROC curve of TG/
HDL-c to detect GDM was 0.617 (95\% CI, 0.548-0.686), and when the TG/HDL-c combined with $\mathrm{HbA1c}$, the area under the combined ROC curve could be improved to 0.705 (95\% CI, 0.637-0.772), suggesting that the sensitivity and specificity of TG/HDL-c in predicting GDM risk could be improved by the concurrent measurement of $\mathrm{HbAlc}$ in pregnant women. Although we were unable to perform the similar analyses due to the lack of data on $\mathrm{HbAlc}$, our study also found the potential of TG/HDL-c for detecting the risk of GDM with an AUC of 0.664 (0.595-0.733), a result which is consistent with the previous one. ${ }^{29}$ Interestingly, Santos-Weis et al have suggested the logarithm of TG/HDL-c ratio in early pregnancy is a valuable index to identify pregnant women with low risk of GDM before 24 weeks of gestation. ${ }^{43}$

In addition, fasting glucose level in early pregnancy is also an accurate indicator for the prediction of GDM. ${ }^{44}$ A recent study has confirmed that the risk of GDM can be predicted by the combination of maternal age, prepregnancy BMI, FPG and TG during 8-20 weeks of gestation with an AUC of 0.766 (95\% CI 0.731-0.801). ${ }^{45}$ In our study, we did analyse an index combined TG and FPG-the 
TyG index, which has been reported to be closely associated with insulin resistance in general population, ${ }^{24-26}$ as well as in pregnancies. ${ }^{28}$ Furthermore, a recent study has reported that the relative risk of GDM and LGA infants in women in the top levels ( $>$ 66.6th percentile) of TyG index among the study population was 4.9-folds and 5.3-folds of its risk in women in the bottom levels. ${ }^{30}$ Likewise, we also found women in the highest tertile of TyG index had a significantly higher risk of developing GDM and delivering LGA infants than women in the lowest tertile in this study.

Since HOMA-IR is a recognized indicator of insulin resistance, the predictive ability of HOMA-IR to GDM was also analysed using ROC curve analysis with the purpose of comparing it with the two TG-associated indices. To be noted, the AUC value and $95 \%$ CI for TyG index to detect GDM was almost the same as HOMA-IR, which suggests TyG index is a good indicator of insulin resistance in early pregnancy. ${ }^{28}$

Furthermore, we compared the ability of TG/HDL-c and TyG index to predict the risk of GDM and LGA infant delivery with the purpose of finding a better index to predict later pregnancy complications in early period of pregnancy. However, we found that there were no statistical differences among them in the ability of predicting the risk of GDM or LGA infant.

In addition to the above, it is important to note that the associations between the two TG-associated indices and the risk of LGA infant became non-significant when GDM status was further adjusted as a cofounding factor following other covariates (Supplemental Table), which is inconsistent with a more recent study in which maternal lipids are significantly associated with newborn adiposity independent of GDM status. ${ }^{46}$ The potential explanation for this discrepancy may be due to the different points of time in the measurement of serum lipid profile (including TG, TC, HDL-c and LDL-c), whether there are other reasons remains to be further investigated.

\section{Strengths and Limitations}

The strengths of our study are the prospective design, as well as its careful recording of obstetric complications by researchers who were blind to the serum lipids of the participants. In consistence with previous studies, ${ }^{28-30}$ this study confirmed the ability of TG/HDL-c and TyG index to predict the risk of later GDM and LGA infant delivery. As well, blood samples were collected at the first trimester of pregnancy, which may provide earlier data support and more time for intervention to prevent or reduce the occurrence of pregnancy complications. On the other side, our study has several limitations that should be noted. First, we did not collect adequate information regarding dietary factors which could act as potential confounders. Second, we did not consider other variables such as a previous history of LGA infant delivery.

\section{Conclusions}

In conclusion, this preliminary cohort study determined that maternal first-trimester TG/HDL-c and TyG could be useful in predicting later development of GDM and LGA infant in Chinese pregnant women. Further, this study confirmed that there is no difference between the two indices in the prediction of GDM and LGA infant delivery. Therefore, they can be used as alternative indices in evaluation the risk of complications during pregnancy and perinatal period.

\section{Acknowledgment}

We thank We thank Shanghai GeneX Biotech Co., Ltd. for their free technical support, and we also acknowledge $\mathrm{Li}$ ShanShan, Wang Bingxin, Li Baolei, Li Rui, Luo Haoze, Zhou Zikun, and Bao Yuanyuan who helped with collecting the anonymous data from routinely collected maternity records.

\section{Author Contributions}

All authors made substantial contributions to conception and design, acquisition of data, or analysis and interpretation of data; took part in drafting the article or revising it critically for important intellectual content; gave final approval of the version to be published; and agree to be accountable for all aspects of the work.

\section{Funding}

This research did not receive any specific grant from funding agencies in the public, commercial, or not-forprofit sectors.

\section{Disclosure}

The authors declare no conflicts of interest.

\section{References}

1. McIntyre HD, Catalano P, Zhang C, Desoye G, Mathiesen ER, Damm P. Gestational diabetes mellitus. Nat Rev Dis Primers. 2019;5 (1):47. doi:10.1038/s41572-019-0098-8 
2. Gao C, Sun X, Lu L, Liu F, Yuan J. Prevalence of gestational diabetes mellitus in mainland China: a systematic review and meta-analysis. J Diabetes Investig. 2019;10(1):154-162. doi:10.1111/jdi.12854

3. The Expert Committee on the Diagnosis and Classification of Diabetes Mellitus. Report of the expert committee on the diagnosis and classification of diabetes mellitus. Diabetes Care. 1997;20:1183-1197. doi:10.2337/diacare.20.7.1183

4. American Diabetes Association. Diagnosis and classification of diabetes mellitus. Diabetes Care. 2015;38(Suppl 1):S8-S16. doi:10.233 7/dc15-S005

5. Hod M, Kapur A, Sacks DA, et al. The International Federation of Gynecology and Obstetrics (FIGO) Initiative on gestational diabetes mellitus: a pragmatic guide for diagnosis, management, and care. Int J Gynaecol Obstet. 2015;131(Suppl 3):S173-S211. doi:10.1016/ S0020-7292(15)30033-3

6. Schiavone M, Putoto G, Laterza F, Pizzol D. Gestational diabetes: an overview with attention for developing countries. Endocr Regul. 2016;50:62-71. doi:10.1515/enr-2016-0010

7. Farrar D, Simmonds M, Bryant M, et al. Hyperglycaemia and risk of adverse perinatal outcomes: systematic review and meta-analysis. BMJ. 2016;354:i4694. doi:10.1136/bmj.i4694

8. Fadl HE, Ostlund IK, Magnuson AF, Hanson US. Maternal and neonatal outcomes and time trends of gestational diabetes mellitus in Sweden from 1991 to 2003. Diabet Med. 2010;27:436-441. doi:10.1111/j.1464-5491.2010.02978.x

9. Lowe WL Jr, Scholtens DM, Kuang A, et al. Hyperglycemia and adverse pregnancy outcome follow-up study (HAPO FUS): maternal gestational diabetes mellitus and childhood glucose metabolism. Diabetes Care. 2019;42:372-380. doi:10.2337/dc18-1646

10. Di Cianni G, Lacaria E, Lencioni C, Resi V. Preventing type 2 diabetes and cardiovascular disease in women with gestational diabetes - the evidence and potential strategies. Diabetes Res Clin Pract. 2018;145:184-192.

11. Mouzon SH, Lassance L. Endocrine and metabolic adaptations to pregnancy; impact of obesity. Horm Mol Biol Clin Investig. 2015;24:65-72. doi:10.1515/hmbci-2015-0042

12. Zeng Z, Liu F, Li S. Metabolic adaptations in pregnancy: a review. Ann Nutr Metab. 2017;70:59-65. doi:10.1159/000459633

13. Herrera E. Lipid metabolism in pregnancy and its consequences in the fetus and newborn. Endocrine. 2002;19:43-55. doi:10.1385/ ENDO: 19:1:43

14. Villar J, Cogswell M, Kestler E, Castillo P, Menendez R, Repke JT. Effect of fat and fat-free mass deposition during pregnancy on birth weight. Am J Obstet Gynecol. 1992;167:1344-1352. doi:10.1016/ S0002-9378(11)91714-1

15. Wiznitzer A, Mayer A, Novack V, et al. Association of lipid levels during gestation with preeclampsia and gestational diabetes mellitus: a population-based study. Am J Obstet Gynecol. 2009;201:482.e1-e8. doi:10.1016/j.ajog.2009.05.032

16. Farias DR, Franco-Sena AB, Vilela A, Lepsch J, Mendes RH, Kac G. Lipid changes throughout pregnancy according to pre-pregnancy BMI: results from a prospective cohort. BJOG. 2016;123:570-578. doi:10.1111/1471-0528.13293

17. Liang Z, Wu Y, Zhu X, Fang Q, Chen D. Insulin resistance and lipid profile during an oral glucose tolerance test in women with and without gestational diabetes mellitus. J Obstet Gynaecol. 2016;36:337-339. doi:10.3109/01443615.2015.1060197

18. Li G, Kong L, Zhang L, et al. Early pregnancy maternal lipid profiles and the risk of gestational diabetes mellitus stratified for body mass index. Reprod Sci. 2015;22:712-717. doi:10.1177/1933719114557896

19. Korkmazer E, Solak N. Correlation between inflammatory markers and insulin resistance in pregnancy. $J$ Obstet Gynaecol. 2015;35:142-145. doi:10.3109/01443615.2014.948408

20. Ryckman KK, Spracklen CN, Smith CJ, Robinson JG, Saftlas AF. Maternal lipid levels during pregnancy and gestational diabetes: a systematic review and meta-analysis. BJOG. 2015;122:643-651. doi:10.1111/1471-0528.13261
21. Ghodke B, Pusukuru R, Mehta V. Association of lipid profile in pregnancy with preeclampsia, gestational diabetes mellitus, and preterm delivery. Cureus. 2017;9:e1420.

22. Zhu WW, Yang HX, Wei YM, et al. Evaluation of the value of fasting plasma glucose in the first prenatal visit to diagnose gestational diabetes mellitus in china. Diabetes Care. 2013;36:586-590. doi: $10.2337 / \mathrm{dc} 12-1157$

23. Hao M, Lin L. Fasting plasma glucose and body mass index during the first trimester of pregnancy as predictors of gestational diabetes mellitus in a Chinese population. Endocr J. 2017;64:561-569. doi:10.1507/endocrj.EJ16-0359

24. Gasevic D, Frohlich J, Mancini GB, Lear SA. The association between triglyceride to high-density-lipoprotein cholesterol ratio and insulin resistance in a multiethnic primary prevention cohort. Metabolism. 2012;61:583-589. doi:10.1016/j.metabol.2011.09.009

25. Fan X, Liu EY, Hoffman VP, Potts AJ, Sharma B, Henderson DC. Triglyceride/high-density lipoprotein cholesterol ratio: a surrogate to predict insulin resistance and low-density lipoprotein cholesterol particle size in nondiabetic patients with schizophrenia. J Clin Psychiatry. 2011;72:806-812. doi:10.4088/JCP.09m05107yel

26. Won KB, Park GM, Lee SE, et al. Relationship of insulin resistance estimated by triglyceride glucose index to arterial stiffness. Lipids Health Dis. 2018;17:268. doi:10.1186/s12944-018-0914-2

27. Rodriguez-Moran M, Simental-Mendia LE, Guerrero-Romero F. The triglyceride and glucose index is useful for recognising insulin resistance in children. Acta Paediatr. 2017;106:979-983. doi:10.1111/ apa.13789

28. Poveda NE, Garces MF, Darghan AE, et al. Triglycerides/glucose and triglyceride/high-density lipoprotein cholesterol indices in normal and preeclamptic pregnancies: a longitudinal study. Int J Endocrinol. 2018;2018:8956404. doi:10.1155/2018/8956404

29. Wang D, Xu S, Chen H, Zhong L, Wang Z. The associations between triglyceride to high-density lipoprotein cholesterol ratios and the risks of gestational diabetes mellitus and large-for-gestational-age infant. Clin Endocrinol (Oxf). 2015;83:490-497. doi:10.1111/cen.12742

30. Pazhohan A, Rezaee Moradali M, Pazhohan N. Association of first-trimester maternal lipid profiles and triglyceride-glucose index with the risk of gestational diabetes mellitus and large for gestational age newborn. J Matern Fetal Neonatal Med. 2019;32:1167-1175. doi:10.1080/14767058.2017.1402876

31. Metzger BE, Gabbe SG, Persson B, et al. International association of diabetes and pregnancy study groups recommendations on the diagnosis and classification of hyperglycemia in pregnancy. Diabetes Care. 2010;33(3):676-682. doi:10.2337/dc10-0719

32. Gong XM, Li ZH, Yu RJ. Maternal and fetal general parameters. In: Zhang WY, editor. Chinese Perinatology. Beijing: People's Medical Publishing House; 2012:1592-1593.

33. Simental-Mendia LE, Rodriguez-Moran M, Guerrero-Romero F. The product of fasting glucose and triglycerides as surrogate for identifying insulin resistance in apparently healthy subjects. Metab Syndr Relat Disord. 2008;6:299-304. doi:10.1089/met.2008.0034

34. DeLong ER, DeLong DM, Clarke-Pearson DL. Comparing the areas under two or more correlated receiver operating characteristic curves: a nonparametric approach. Biometrics. 1988;8:37-45.

35. Enquobahrie DA, Williams MA, Qiu C, Luthy DA. Early pregnancy lipid concentrations and the risk of gestational diabetes mellitus. Diabetes Res Clin Pract. 2005;70:134-142. doi:10.1016/j. diabres.2005.03.022

36. Schaefer-Graf UM, Graf K, Kulbacka I, et al. Maternal lipids as strong determinants of fetal environment and growth in pregnancies with gestational diabetes mellitus. Diabetes Care. 2008;31:1858e63. doi: $10.2337 / \mathrm{dc} 08-0039$

37. Schaefer-Graf UM, Kjos SL, Kilavuz O, et al. Determinants of fetal growth at different periods of pregnancies complicated by gestational diabetes mellitus or impaired glucose tolerance. Diabetes Care. 2003;26:193e8. doi:10.2337/diacare.26.1.193 
38. Di Cianni G, Miccoli R, Volpe L, et al. Maternal triglyceride levels and newborn weight in pregnant women with normal glucose tolerance. Diabet Med. 2005;22:21e5. doi:10.1111/j.1464-5491.2004.01336.x

39. Son GH, Kwon JY, Kim YH, Park YW. Maternal serum triglycerides as predictive factors for large-for-gestational age newborns in women with gestational diabetes mellitus. Acta Obstet Gynecol Scand. 2010;89:700e4. doi:10.3109/00016341003605677

40. Hashemipour S, Haji Seidjavadi E, Maleki F, Esmailzadehha N, Movahed F, Yazdi Z. Level of maternal triglycerides is a predictor of fetal macrosomia in non-obese pregnant women with gestational diabetes mellitus. Pediatr Neonatol. 2018;59(6):567-572. doi:10.1016/j. pedneo.2018.01.008

41. Herrera E, Ortega-Senovilla H. Disturbances in lipid metabolism in diabetic pregnancy - are these the cause of the problem? Best Pract Res Clin Endocrinol Metab. 2010;24:515e25. doi:10.1016/j.beem.2010.05.006

42. Jin WY, Lin SL, Hou RL, et al. Associations between maternal lipid profile and pregnancy complications and perinatal outcomes: a population-based study from China. BMC Pregnancy Childbirth. 2016;16:60. doi:10.1186/s12884-016-0852-9
43. Dos Santos-Weiss IC, Rea RR, Fadel-Picheth CM, et al. The plasma logarithm of the triglyceride/HDL-cholesterol ratio is a predictor of low risk gestational diabetes in early pregnancy. Clin Chim Acta. 2013;418:1-4. doi:10.1016/j.cca.2012.12.004

44. Wang C, Zhu W, Wei Y, et al. The predictive effects of early pregnancy lipid profiles and fasting glucose on the risk of gestational diabetes mellitus stratified by body mass index. J Diabetes Res. 2016;2016:3013567. doi:10.1155/2016/3013567

45. Zheng T, Ye W, Wang X, et al. A simple model to predict risk of gestational diabetes mellitus from 8 to 20 weeks of gestation in Chinese women. BMC Pregnancy Childbirth. 2019;19:252. doi:10.1186/s12884-019-2374-8

46. Samsuddin S, Arumugam PA, Md Amin MS, et al. Maternal lipids are associated with newborn adiposity, independent of GDM status, obesity and insulin resistance: a prospective observational cohort study. BJOG. 2020;127:490-499. doi:10.1111/1471-0528.16031

Diabetes, Metabolic Syndrome and Obesity: Targets and Therapy

Dovepress

\section{Publish your work in this journal}

Diabetes, Metabolic Syndrome and Obesity: Targets and Therapy is an international, peer-reviewed open-access journal committed to the rapid publication of the latest laboratory and clinical findings in the fields of diabetes, metabolic syndrome and obesity research. Original research, review, case reports, hypothesis formation, expert opinion and commentaries are all considered for publication. The manuscript management system is completely online and includes a very quick and fair peer-review system, which is all easy to use. Visit http://www.dovepress.com/testimonials.php to read real quotes from published authors.

Submit your manuscript here: https://www.dovepress.com/diabetes-metabolic-syndrome-and-obesity-targets-and-therapy-journal 